\title{
Brain death: medical management in seven Brazilian pediatric intensive care units
}

\author{
Patricia M. Lago, 1 Jefferson Piva, ${ }^{2}$ Pedro Celiny Garcia, ${ }^{3}$ Eduardo Troster, 4 \\ Albert Bousso, ${ }^{5}$ Maria Olivia Sarno, 6 Lara Torreão, 7 Roberto Sapolnik; 8 \\ Members of Núcleo de Estudos em Ética em Pediatria, Brazil (NEEP-Br) 9
}

\begin{abstract}
Objective: To assess the incidence of brain death (BD) and its medical management and adopted protocols after its diagnosis in seven pediatric intensive care units (PICUs) located in three Brazilian regions.

Methods: A cross-sectional and multicenter study was conducted, based on the retrospective review of medical records regarding all deaths that occurred between January 2003 and December 2004 in seven Brazilian PICUs of tertiary hospitals located in Porto Alegre (two), São Paulo (two) and Salvador (three). Two pediatric intensive care residents from each hospital were previously trained and filled out a standard protocol for the investigation of demographic data, cause of death, diagnosis of $\mathrm{BD}$, related protocols and subsequent medical management.

Results: A total of 525 death patients were identified and $61(11.6 \%)$ were defined as BD. The incidence of BD was different $(p=0.015)$ across the seven PICUs, but with no difference across the three regions. Intracranial hemorrhage was the most frequent cause of $\mathrm{BD}(31.1 \%)$. In $80 \%$ of the cases the diagnosis of BD was confirmed by complementary exams ( south $=100 \%$, southeast $=68 \%$ and northeast $=72 \% ; p=0.02$ ). The interval between the diagnosis of BD and the withdrawal of life support was different $(p<0.01)$ across the three regions, being faster $(p=0.04)$ in the south $(1.8 \pm 1.9 \mathrm{~h})$ than in the southeast $(28.6 \pm 43.2 \mathrm{~h})$ and than in the northeast $(15.5 \pm 17.1 \mathrm{~h})$. Only six $(9.8 \%)$ children with BD were organ donors.
\end{abstract}

Conclusion: Although a Brazilian law defining the criteria for the determination of BD has been in place since 1997, we verified that it is not followed as strictly as it should be. Consequently, unnecessary life support is offered to deceased individuals, and there is a discrete involvement of PICUs in organ donation.

J Pediatr (Rio J). 2007;83(2):133-140: Brain death, organ donation, pediatric intensive care, medical ethics.

1. Pediatra intensivista, Unidade de Tratamento Intensivo Pediátrico (UTIP), Hospital de Clínicas de Porto Alegre (HCPA), Porto Alegre, RS. Doutoranda, Programa de Pós-Graduação em Pediatria e Saúde da Criança, Faculdade de Medicina, Pontifícia Universidade Católica do Rio Grande do Sul (PUCRS), Porto Alegre, RS, Brasil.

2. Professor adjunto, Departamento de Pediatria, PUCRS e Universidade Federal do Rio Grande do Sul (UFRGS), Porto Alegre, RS. Chefe associado, UTIP, Hospital São Lucas, PUCRS, Porto Alegre, RS, Brasil.

3. Professor adjunto, Departamento de Pediatria, PUCRS, Porto Alegre, RS. Chefe associado, UTIP, Hospital São Lucas, PUCRS, Porto Alegre, RS, Brasil.

4. Professor adjunto, Departamento de Pediatria, Universidade de São Paulo (USP), São Paulo, SP. Chefe, UTIP, Instituto da Criança, USP, São Paulo, SP, Brasil.

5. Chefe, UTIP, Hospital Universitário, USP, São Paulo, SP. Médico assistente, UTIP, Instituto da Criança, USP, São Paulo, SP, Brasil.

6. Pediatra intensivista e chefe, UTIP, Hospital da Criança de Salvador, Salvador, BA, Brasil.

7. Professora, Departamento de Pediatria, Universidade Federal da Bahia (UFBA), Salvador, BA. Pediatra intensivista e chefe, UTIP, Hospital Ernesto Simões Filho, Salvador, BA, Brasil.

8. Pediatra intensivista e chefe, UTIP, Hospital São Rafael de Salvador, Salvador, BA, Brasil.

9. Members of Núcleo de Estudos em Ética em Pediatria, Brazil (NEEP-Br).

Financial support: CNPq, protocol no. 054/2005.

Manuscript received Aug 15 2006, accepted for publication Nov 102006.

Suggested citation: Lago PM, Piva J, Garcia PC, Troster E, Bousso A, Sarno MO, et al. Brain death: medical management in seven Brazilian pediatric intensive care units. J Pediatr (Rio J). 2007;83(2):133-140.

doi 10.2223/JPED.1594 


\section{Introduction}

Before the advent of mechanical ventilation, death was defined by the loss of circulatory, respiratory or neurological function. Nowadays, owing to the improvements in cardiorespiratory support, it is possible to maintain vital functions for long time periods, even in the absence of brain activity. This perspective raises at least two major questions: to what extent should a patient be considered alive? and Is organ donation possible in individuals under perfusion and whose heart is still beating ${ }^{1}$

In an attempt to overcome this problem, a president's commission was established in the United States in 1981, which defined death as the irreversible cessation of circulatory and respiratory functions or irreversible loss of full brain function. ${ }^{1,2}$

Brain death (BD) is currently defined as the loss of full brain function, characterized by deep coma, apnea and lack of supraspinal reflexes. Therefore, BD according to medical and ethical aspects corresponds to the death of an individual, since the brain is essential for the whole body function. ${ }^{3,4}$

The diagnosis of BD is clinical. In some countries (e.g.: United States), confirmation of BD at bedside is enough. ${ }^{4}$ However, other countries recommend examinations that confirm the absence of electrical and metabolic functions or of cerebral flow. Electroencephalogram (EEG) is the most widely used exam in several countries. Nevertheless, this exam cannot be easily performed in the intensive care unit (ICU), due to the possible interference of artifacts with electrical activity. ${ }^{4}$ It has been shown that $20 \%$ of patients with BD may have some EEG activity. ${ }^{5}$ Cerebral ultrasound has a sensitivity of $90 \%$ and a specificity of $100 \%$, and is performed on middle and vertebral cerebral arteries.

In Brazil, the National Medical Council (Conselho Federal de Medicina, CFM) published the diagnostic criteria for BD in 1997. The clinical criteria must be described in the declaration of $\mathrm{BD}$ by two experienced physicians, not necessarily neurologists, at an interval that varies according to the age of each patient. Complementary exams that confirm total absence of brain function are mandatory. ${ }^{6}$ This rule facilitates organ donation, and when this is not possible, it allows removing those patients considered to be dead from the life support system.

The criteria for BD are accepted by most western countries, but some physicians from the Latin American community are still reluctant to remove ventilatory support from patients whose heart is still beating. This difficulty increases the conflict between the medical staff and family members, especially when personal or religious values are involved. Some cultures and religious groups do not accept death if vital functions have not ceased. In these cases, the removal of life support causes discomfort, since the individual is apparently alive because his/her body is being kept through an artificial support system. ${ }^{6,7}$

In Brazil, the declaration of BD to organ donation teams is mandatory. ${ }^{8}$ If donation is not possible, the physician must withdraw life support. Withdrawal of inotropic agents and of mechanical ventilation is legally supported and should be done by the intensivist after the family is contacted. ${ }^{5}$

Even with well-defined criteria for over 2 decades, the diagnosis of $\mathrm{BD}$ and its subsequent management are still controversial and cause anxiety in the medical community all over the world. ${ }^{6,7,9}$ Few studies have been published on BD in children. ${ }^{10-12}$ We believe that, in Brazil, just as in other countries, medical management regarding BD is not uniform, with regional discrepancies. The aim of this study is to assess the reality of $B D$ in Brazilian pediatric intensive care units (PICU), its incidence, adherence to the protocol established by the CFM and the management used in the PICUs of three Brazilian regions.

\section{Methods}

An observational, cross-sectional and retrospective study was carried out in seven PICUs with all children who died between 2003 and 2004 and who had been diagnosed with BD in their medical records. Seven PICUs were selected for this study:

a) two in the Southern region - Hospital São Lucas, affiliated with Pontifícia Universidade Católica do Rio Grande do Sul (PUCRS) and Hospital de Clínicas de Porto Alegre (HCPA), affiliated with Universidade Federal do Rio Grande do Sul (UFRGS);

b) two in the Southeastern region - Hospital das Clínicas de São Paulo and Hospital Universitário, both affiliated with Universidade de São Paulo (USP);

c) three in the Northeastern region - Hospital São Rafael, Hospital da Criança and Hospital Ernesto Simões Filho.

These seven PICUs operate in a similar fashion, with professors or coordinators who visit them every day, in addition to hired physicians and residents in pediatrics and/or intensive therapy that care for the patients during the day and night. The management of each patient is defined by the whole medical staff in daily meetings. All PICUs assessed perform extremely complex procedures. The Southern and Southeastern region hospitals carry out organ transplants.

Both patients and their medical records were identified by active search, by reviewing all admission and discharge data of each PICU between January 1, 2003 and December 31, 2004. The medical records were then carefully reviewed in search of patients who had the diagnosis of BD established according to their daily outcome, prior to their death. Two physicians from each PICU were selected and trained for data collection. They filled out a protocol with objective answers 
using the data obtained from the patients' medical records. If any questions arose, they were discussed over the phone or via Internet.

From the selected medical records, the following information was obtained:

a) demographic and general data about each patient;

b) data about the death (number of multiple organ failures in the last $48 \mathrm{~h}$ of life, date and time of death);

c) data related to $\mathrm{BD}$ (date and time of BD diagnosis, complementary exams for the diagnosis, necessity for complete cardiopulmonary resuscitation, dose of vasopressor drugs at $48 \mathrm{~h}, 24 \mathrm{~h}$ and at the time of death, as well as mechanical ventilation parameters at $48 \mathrm{~h}, 24 \mathrm{~h}$ and at the time of death;

d) organ donation.

Based on the admission and death data obtained from each PICU in the last years, we estimated that each unit had approximately 400 admissions a year with a mortality rate of $10 \%$, amounting to nearly 560 deaths in 2 years. Based on studies carried out in PICUs in southern Brazil and in Argentina, ${ }^{10-12}$ the $\mathrm{BD}$ rate for this group is estimated at $10 \%$. Therefore, we expected to obtain approximately 56 cases of BD in these seven PICUs.

The continuous variables were expressed as mean and standard deviation, and those without normal distribution were expressed as median and interquartile range (IQR $25-75 \%)$. Student's $t$ test and ANOVA with Bonferroni's post hoc test, in case of differences between three or more groups, were used to compare normally-distributed continuous variables. The Kruskal-Wallis and Mann-Whitney tests were used to compare variables without normal distribution. The post hoc analysis of data with asymmetric distribution was made using rank ANOVA for asymmetric data and using Tukey's post hoc test. The categorical variables were expressed as percentage and compared by the chi-square test and by Fisher's exact test, followed by the Finner-Bonferroni's p-value adjustment. All the data were loaded into an Excel (Microsoft) spreadsheet and analyzed by SPSS 11.0.

The present study was approved by the Research Ethics Committees of the seven hospitals that participated in the study.

\section{Results}

In the seven PICUs analyzed, there were 525 deaths in 2003 and 2004, 152 of which occurred in two PICUs in the southern region, 143 in two PICUs in the southeastern region and 230 in three PICUs in the northeastern region.

In 61 of 525 deaths (11.6\%), the diagnosis of BD was identified in the medical records. The incidence of BD in these seven PICUs ranged from 4.5 to $24.2 \%(p=0.015)$ (Table 1 ). However, when we grouped and compared the deaths per region (Table 2), we did not find any difference in the incidence of $\mathrm{BD}$ across the three regions (7.4, 15.3 and $12.6 \% ; p=0.052)$. According to Table 2 , the general characteristics (sex, age and number of organ failures) of this group with diagnosis of BD did not differ across the three regions.

Table 1 - Distribution of deaths and the respective incidence of brain death in seven pediatric intensive care units*

\begin{tabular}{lcccc}
\hline Hospital & No. of deaths & Deaths $<\mathbf{2 4} \mathbf{h}$ & $\mathbf{B D}, \mathbf{n}(\mathbf{\%})$ & Deaths due to other causes \\
\hline 1 & 67 & 4 & & 57 \\
2 & 85 & 6 & $6(8.9)$ & 64 \\
3 & 110 & 9 & $15(17.6)$ & 87 \\
4 & 33 & 1 & $14(12.7)$ & 24 \\
5 & 101 & 4 & $8(24.2)$ & 91 \\
6 & 67 & 8 & $6(5.9)$ & 56 \\
7 & 62 & 4 & $3(4.5)$ & 49 \\
Total & 525 & 36 & $9(14.5)$ & 428 \\
\end{tabular}

$\mathrm{BD}=$ brain death. $* \mathrm{p}=0.015$ (Fisher's exact test). 
The length of stay in the PICU prior to death varied considerably and significantly across the seven hospitals (2 to 455 days, $p=0.041)$. However, this difference was not observed when the medians for PICU stay were compared across the three regions ( 2 to 5 days, $p=0.12$ ).

In $80.3 \%$ of cases, the clinical diagnosis of $\mathrm{BD}$ was confirmed by brain flow tests (ultrasound or scintigraphy) and/or EEG. The southern region was the one that best complied with the recommendations for definition of BD in
Brazil (confirmation through complementary exams), where $100 \%$ of patients with BD underwent confirmatory tests, comparatively to $68 \%$ in the southeastern region and to $72 \%$ in the northeastern region ( $p=0.02)$.

Cerebrovascular accident was the most frequent cause ( $31.1 \%)$ of $\mathrm{BD}$, followed by post-cardiorespiratory arrest and meningoencephalitis (Table 3).

Out of 61 patients diagnosed with BD, only six (9.8\%) were organ donors (four patients in the southern region and

Table 2 - Demographic characteristics of deaths resulting from brain death in seven pediatric intensive care units in three Brazilian regions

\begin{tabular}{|c|c|c|c|c|}
\hline & $\begin{array}{c}\text { South } \\
\mathrm{n}=167 \text { ( } 2 \text { PICUs })\end{array}$ & $\begin{array}{l}\text { Southeast } \\
\mathrm{n}=143(2 \text { PICUs })\end{array}$ & $\begin{array}{c}\text { Northeast } \\
\mathrm{n}=230 \text { ( } 3 \text { PICUs })\end{array}$ & $\mathbf{p}$ \\
\hline Brain death: $\mathrm{n}(\%)$ & $21(12.6)$ & $22(15.3)$ & $18(7.4)$ & 0.052 \\
\hline \multicolumn{5}{|l|}{ Age (months) } \\
\hline Mean \pm SD & $62.6 \pm 58.8$ & $60.3 \pm 55.8$ & $81.6 \pm 58.6$ & 0.48 \\
\hline Median IQR 25-75\% & $46(9-115)$ & $39.5(11-97)$ & $73.5(28-135.7)$ & 0.34 \\
\hline Male sex $(\%)$ & $13(59)$ & $10(45)$ & $7(39)$ & 0.32 \\
\hline \multicolumn{5}{|l|}{ L in the PICU (days) } \\
\hline Mean \pm SD & $6.7 \pm 7.1$ & $27.2 \pm 95.7$ & $5.5 \pm 6.5$ & 0.48 \\
\hline Median IQR 25-75\% & $2(5-7)$ & $5(3.2-8.7)$ & $3(2-5.7)$ & 0.12 \\
\hline More than 3 organ failures (\%) & $11(52)$ & $10(45)$ & $14(77)$ & 0.10 \\
\hline
\end{tabular}

Table 3 - Causes of brain death in seven pediatric intensive care units in three Brazilian regions

\begin{tabular}{|c|c|c|c|c|c|c|c|}
\hline & PCRA & CVA & Mgtis/Enc & HT and ICH & Tumor & Snake & Total \\
\hline South & 3 & 5 & 7 & 4 & 2 & 0 & 21 \\
\hline Southeast & 7 & 7 & 2 & 5 & 1 & 0 & 22 \\
\hline Northeast & 1 & 7 & 4 & 4 & 1 & 1 & 18 \\
\hline Donors & 3 & 1 & 0 & 2 & 0 & 0 & 6 \\
\hline
\end{tabular}

$\mathrm{CVA}=$ cerebrovascular accident; $\mathrm{HT}=$ head trauma; $\mathrm{ICH}=$ intracranial hypertension; $\mathrm{Mgtis} / \mathrm{Enc}=$ meningitis and/or encephalitis; PCRA = post-cardiorespiratory arrest; Snake = snakebite. 
two in the southeastern region, $p=0.13$ ). Postcardiorespiratory arrest was the most frequent cause of BD in organ donors (three cases). Of 13 deaths due to head trauma and/or intracranial hypertension, situation that favors organ donation, there were only two donations (Table 3). When we compared organ donors and non-donors, we did not find any significant difference in age $(p=0.56)$ or in the length of stay in the PICU $(p=0.24)$.

The time between diagnosis of BD and withdrawal of life support was different across the seven PICUs, ranging from 1 to $193 \mathrm{~h}(\mathrm{p}=0.01)$. This difference was observed again when we grouped and compared the three regions. We noted that the average time for withdrawal of life support after the diagnosis of BD was significantly $(p=0.041)$ lower in the southern region $(1.8 \pm 1.9 \mathrm{~h})$ than in the southeastern and northeastern regions $(28.6 \pm 43.2 \mathrm{~h}$ and $15.5 \pm 17.1 \mathrm{~h}$, respectively). In the southern region, no patient with $\mathrm{BD}$ was kept for over $24 \mathrm{~h}$ on the life support system, differently ( $p=0.002$ ) from the northeastern and southeastern regions, where ventilatory support was kept for more than $24 \mathrm{~h}$ in 17 patients ( $~ 40 \%$ of BD in these regions), but none of them was an organ donor. In $13(21 \%)$ patients, we found an increase in the use of inotropic agents after the diagnosis of $\mathrm{BD}$, and in this case, only six patients were organ donors. In 28 patients, respiratory frequency of the ventilatory support was maintained or increased after the diagnosis, with differences across the three regions $(p<0.001)$ (Table 4$)$.

\section{Discussion}

From the scientific and ethical point of view, BD certainly corresponds to the death of an individual. In the United States, the criteria that define BD were published in $1981,{ }^{1}$ whereas in Brazil, these criteria were legally adopted in 1997. ${ }^{6}$ The necessity to establish criteria that define BD and to legalize them aims to encourage and standardize organ donation, and allow for the removal of life support from nondonor patients in irreversible coma. ${ }^{5}$ The concept of BD should convey the idea of what being alive or dead means, not being directly related to organ donation. ${ }^{12}$

By analyzing the diagnosis of $\mathrm{BD}$ and the medical management subsequently adopted in seven PICUs in three different Brazilian regions, we note that this issue is not sufficiently clear among Brazilian pediatric intensivists, with regional variations and without uniform actions. Therefore, in this study, we may point out that:

a) the incidence of BD varied considerably across hospitals;

b) although the Brazilian law is clear as to the necessity of complementary exams for the diagnosis of BD, such exams were not carried out in $20 \%$ of the cases diagnosed as BD;
C) the time for removal of life support after the diagnosis of $\mathrm{BD}$ was extremely long. In the southeastern and northeastern regions, over $40 \%$ of children diagnosed with BD were kept for over $24 \mathrm{~h}$ on ventilatory support;

d) organ donation rates were not significant.

The incidence of BD in adult ICUs in Europe and in the United States amounts to approximately $12 \%,{ }^{13}$ which is similar to the one described in studies undertaken in PICUs of Brazil, Argentina and United States. ${ }^{10,11,14}$ In this study, the overall incidence of $\mathrm{BD}(11.6 \%)$ was similar to the one described in the literature. However, when the hospitals were analyzed separately, there was a remarkable difference, which ranged from $4.5 \%$ in one hospital in the northeastern region to $24.5 \%$ in a PICU in the southeastern region, in Brazil. This difference can be related to the characteristics of inpatients, or be related to the difficulty in establishing the diagnosis of BD due to medical or technical problems in some of these Brazilian hospitals. ${ }^{13,15}$

The clinical confirmation of BD requires deep coma with absence of supraspinal activity and apnea secondary to an irreversible process of known etiology. ${ }^{15}$ In Brazil, as well as in several European, Asian, Central American and South American countries, the diagnosis of $\mathrm{BD}$ requires confirmatory tests, showing absence of electrical and metabolic brain activity or of blood perfusion. This situation differs from that of the United States, for instance, where complementary exams are optional, and where only clinical examination at bedside is enough. In Canada, EEG is not considered to be a confirmatory test and, in Switzerland, brain angiography is mandatory for all patients with clinical diagnosis of $\mathrm{BD} .^{3,4}$

The statement of $\mathrm{BD}$, according to the resolution of CFM in 1997, establishes that confirmatory tests should be performed in donor and in nondonor patients, so that life support can be withdrawn. ${ }^{6}$ In this study, we observed that $20 \%$ of patients diagnosed with BD described in the medical records were not submitted to confirmatory tests. This may be the result of the poor infrastructure for such exams in some hospitals. On the other hand, another hypothesis is the bias of the medical staff in not wanting to cease life support in this situation and, in such case, the exams would not be necessary.

After confirmation of $\mathrm{BD}$, the withdrawal of life support is an ethical duty of the medical staff, since maintaining life support in these cases is not beneficial, in addition to causing suffering to the family and unnecessary expenses.

In order to avoid conflicts, the medical staff may choose to keep life support until cardiac arrest occurs "spontaneously," with a do-not-resuscitate order. After BD, even if all life support is maintained, cardiac arrest occurs in approximately 1 week. ${ }^{1}$ However, there have been some cases in which 
Table 4 - Medical management after the diagnosis of brain death in seven pediatric intensive care units in three Brazilian regions

\begin{tabular}{|c|c|c|c|c|}
\hline & $\begin{array}{c}\text { South } \\
\mathrm{n}=\mathbf{2 1}(2 \text { PICUs })\end{array}$ & $\begin{array}{c}\text { Southeast } \\
\mathrm{n}=\mathbf{2 2}(2 \text { PICUs })\end{array}$ & $\begin{array}{c}\text { Northeast } \\
\mathrm{n}=18 \text { ( } 3 \text { PICUs) }\end{array}$ & $\mathbf{p}$ \\
\hline Time for withdrawal of life support (h) & $1.8 \pm 1.9 *$ & $28.6 \pm 43.2$ & $15.5 \pm 17.1$ & 0.041 \\
\hline Median (minimum-maximum) & $1(1-9) \dagger$ & $20(1-193)$ & $4.5(1-49)$ & 0.002 \\
\hline Withdrawal of MV (\%) & $20(95) \neq$ & $4(18)$ & $3(17)$ & $<0.001$ \\
\hline Increase in the dose of inotropics (\%) & $3(14)$ & $3(14)$ & $7(39)$ & 0.135 \\
\hline $\begin{array}{l}\text { Increase or maintenance of MV } \\
\text { parameters after diagnosis }(\%)\end{array}$ & $0 \neq$ & $16(76)$ & $12(67)$ & $<0.001$ \\
\hline $\begin{array}{l}\text { Maintenance of support for over } \\
24 \mathrm{~h} \text { after diagnosis (\%) }\end{array}$ & $0 \neq$ & $9(41)$ & $8(44)$ & 0.001 \\
\hline Donors (\%) & $4(19)$ & $2(9)$ & 0 & 0.13 \\
\hline
\end{tabular}

$M V$ = mechanical ventilation; PICU = pediatric intensive care unit.

* ANOVA followed by Bonferroni's post hoc test.

+ Rank ANOVA for asymmetric data and Tukey's post hoc test.

* Fisher's exact test followed by Finner-Bonferroni's p-value adjustment.

these patients generated costs and took up ICU beds for nearly 2 months. ${ }^{1}$ The question in this case is about who benefits from the delayed withdrawal of life support from an individual who is already dead. The avoidance of a "possible conflict" does not seem a plausible explanation from the ethical point of view. ${ }^{7}$

Several studies have shown the difficulty pediatric intensivists have in discontinuing therapy in patients with BD. ${ }^{6,12}$ In this study, the time for withdrawal of life support after the diagnosis of $\mathrm{BD}$ differed across the three regions and, in some of them, it was extremely long (more than $24 \mathrm{~h}$ ). In the southeastern and northeastern regions, over $40 \%$ of children were kept on ventilatory support for more than 1 day. Maintenance of life support after confirmation of BD probably results from the lack of information about the concept of $B D$ or from unfounded legal fears.

The CFM admits that physicians have the power to cease life support after confirmation of $B D$, even if the family refuses it, respecting cultural and religious beliefs. ${ }^{5}$ Moreover, in response to the specific request made by PUCRS, ${ }^{16}$ the CFM reaffirms the diagnosis of BD after the clinical criteria are met and complementary exams are performed, and advocates the removal, by physicians, of devices and support that maintain respiratory and cardiocirculatory functions, even in those patients that are not eligible for organ donation.

Although the criteria for BD have been well defined for more than 2 decades, there is still some controversy over this concept. In a recent study, Joffe et al. demonstrated that of 54 pediatric intensivists interviewed in 15 Canadian PICUs, $48 \%$ incorrectly regarded loss of consciousness as diagnosis of BD. In addition, 34\% of interviewees did not feel comfortable switching off the respirator in patients with $B D$ when the family did not authorize the withdrawal of life support. ${ }^{6}$ This behavior suggests that physicians make a distinction between BD and death.

In a study carried out in 16 Argentine PICUs, Althabe also showed the difficulty of pediatric intensivists in accepting $B D$ as death of the patient. This was observed in 52 cases of $B D$, in which ventilatory support was maintained in $100 \%$ and inotropic therapy in $82 \%$ of the cases. ${ }^{12}$ Therefore, there is much that remains to be clarified, discussed and demystified, both in the medical and lay communities.

In regard to organ donation, in this study, we noted a low incidence of donor patients - only six ( $9.8 \%)$, which shows the lack of involvement of medical teams in organ donation initiatives. This lack of commitment of the medical staff analyzed is worrying, since the southern and southeastern 
regions are the major centers for organ transplants in Brazil. Furthermore, patients with BD secondary to head trauma were not donors in any of the hospitals analyzed, which seems to be incoherent, since head trauma is considered one of the major injuries that lead to donation. ${ }^{17-19}$ Morris, for instance, described 40 pediatric patients with head trauma and $\mathrm{BD}$, among whom over $50 \%$ were organ donors, but the main reason for nondonation was the refusal of the family to do so. ${ }^{20}$ In the analyzed patients, we could not check whether some families were asked about organ donation or whether there was no interest in reporting the death to the organ harvest teams.

This study, as most retrospective studies on end-of-life care, has some limitations in terms of methodology. Since this study is based on data from medical records, it is not possible to rule out collection biases. In an attempt to minimize this risk, we developed a protocol with closed and objective questions. Even though we considered these possible methodological biases, we noted that the data obtained are similar to those of other studies on BD carried out in other countries. ${ }^{6,9,19}$

Death-related issues are still taboo in our setting. For this reason, the discussion about data related to $B D$ involving both children and adults and the subsequent management can be the initial step towards an in-depth discussion about ethical and moral aspects regarding end-of-life care, such as death with dignity, responsibility, and correct resource allocation for the management of terminal patients, larger diffusion of transplantation programs, physician-patientfamily relationship based on trust and honesty, and demystification of fantasies. In this study, the difficulty in establishing the diagnosis of $\mathrm{BD}$ and its subsequent management highlights the importance of an urgent change in behavior regarding the end-of-life care of these patients.

\section{Members of Núcleo de Estudos em Ética em Pediatria, Brazil (NEEP-Br)}

Carolina Amoretti, RS, UTIP do Hospital de Clínicas de Porto Alegre; Gleiber Rodrigues, RS, UTIP do Hospital de Clínicas de Porto Alegre; Lisandra Xavier, RS, UTIP do Hospital de Clínicas de Porto Alegre; Graziela de Araújo, SP, UTIP do Instituto da Criança, USP; Débora Oliveira, SP, UTIP do Instituto da Criança, USP; Claudio Fauzine, SP, UTIP do Instituto da Criança, USP; Jose Carlos Fernandes, SP, UTIP do Hospital Universitário, USP; Manuela Borges, BA, Hospital da Criança OSID; Luanda Costa, BA, Hospital da Criança OSID; Carine Junqueira, BA, UTIP do Hospital São Rafael; Valdi Junior, BA; UTIP do Hospital São Rafael; Rodrigo Athanasio, BA, UTIP do Hospital São Rafael; Estela Rocha, BA, UTIP do Hospital São Rafael; Maria Bernadete Lessa, BA, UTIP do Hospital Ernesto Simões Filho.

\section{References}

1. Troug RD, Robinson WM. Role of brain death and the dead-donor rule in the ethics of organ transplantation. Crit Care Med. 2003;31:2391-6.

2. Guidelines for the determination of death. Report of the medical consultants on the diagnosis of death to the President's Commission for the Study of Ethical Problems in Medicine and Biomedical and Behavioral Research. JAMA. 1981;246:2184-6.

3. Lazar NM, Shemie S, Webster GC, Dieckens BM. Bioethics for clinicians: Brain death. CMAJ. 2001;164:833-6.

4. Wijdicks EF. The diagnosis of brain death. N Engl J Med. 2001;344:1215-21.

5. Joffe AR, Anton N. Brain death: understanding of the conceptual basis by pediatric intensivists in Canada. Arch Pediatr Adolesc Med. 2006;160:747-52.

6. Brasil, Conselho Federal de Medicina. Resolução 1480, de 8 de agosto de 1997. http://www.portalmedico.org.br/resolucoes/ cfm/1997/1480_1997.htm. Access: 6/11/2006.

7. Inwald D, Jacobovits I, Petros A. Brain stem death: managing care when accepted medical guidelines and religious beliefs are in conflict. Consideration and promisse are possible. BMJ. $2000 ; 320: 1266-7$.

8. Brasil, Conselho Federal de Medicina. Lei 9434 , de 4 de fevereiro de 1997. http://www.planalto.gov.br/ccivil_03/leis/ L9434.htm. Access: 6/11/2006.

9. Gonzáles NF, Fernández MF, Galán CR, Torre CA, Villanueva MA, Cuervo MS. Muerte encefálica y donación en población infantil. An Pediatr (Barc). 2004;60:450-3.

10. Lago PM, Piva J, Kipper D, Garcia PC, Pretto C, Giongo M, et al. Limitação de suporte de vida em três unidades de terapia intensiva pediátrica do sul do Brasil. J Pediatr (Rio J). 2005;81:111-7.

11. Kipper DJ, Piva JP, Garcia PC, Einloft PR, Bruno F, Lago P, et al. Evolution of the medical practices and modes of death on pediatric intensive care in southern Brazil. Pediatr Crit Care Med. 2005;6:258-63.

12. Althabe M, Cardigni G, Vassalo JC, Allende D, Berrueta M, Codermatz $\mathrm{M}$, et al. Dying in the Intensive Care Unit: collaborative multicenter study about forgoing life-sustaining treatment in Argentine Pediatric Intensive Care Units. Pediatr Crit Care Med. 2003;4:164-9.

13. Senouci K, Guerrini P, Diene E, Atinault A, Claquin J, Bonnet F, et al. A survey on patients admitted in severe coma: implications for brain death identification and organ donation. Intensive Care Med. 2004;30:38-44.

14. Zawistowski CA, DeVita MA. A descriptive study of children dying in the pediatric intensive care unit after withdrawal of lifesustaining treatment. Pediatr Crit Care Med. 2004;5:216-23.

15. Kipper D, Loch J, Piva JP. Dilemas éticos, morais e legais em UTIP. In: Piva J, Garcia P. Medicina intensiva em pediatria. Rio de Janeiro: Revinter; 2005. p. 753-71.

16. Piva JP, Garcia PC, Kipper DJ, Einloft P, Fiori R, Dias F. Resolução sobre morte cerebral em UTI. J Pediatr (Rio J). 1998;74:347-8.

17. Tsai E, Shemie SD, Cox PN, Furst S, McCarthy L, Hebert D. Organ donation in children: role of pediatric intensive care unit. Pediatr Crit Care Med. 2000;1:156-60. 
18. Kompanje EJ, Bakker J, Slicker FJ, Ijzermans JN, Maas AI. Organ donation and unused potential donation in traumatic brain injury, subarachnoid haemorrhage and intracerebral hemorrhage. Intensive Care Med. 2006;32:217-22.

19. Opdam HI, Silvester W. Identifying the potential organ donor: an audit of hospital deaths. Intensive Care Med. 2004;30:1390-7.

20. Morris K, Tasker R, Parslow R, Forsyth R, Hawley C. Organ donation in pediatric traumatic brain injury. Intensive Care Med. $2006 ; 32: 1458$.
Correspondence:

Patrícia Miranda Lago

Rua Furriel Luis Vargas 238/201

CEP 90470130 - Porto Alegre, RS - Brazil

Tel.: /Fax: +55 (51) 3333.4462, +55 (51) 9678.0431

E-mail: lagopatricia@terra.com.br 